\title{
Evaluation of Results of Circumcision Done In Hospital Raja PerempuanZainab II and Circumcision Done In the Community Setting
}

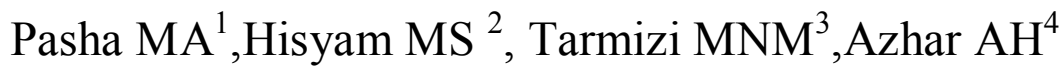 \\ ${ }^{1,4}$ Department of Surgery, School of Medical Sciences, UniversitiSainsMalaysia,KubangKerian,Malaysia \\ ${ }^{2}$ Department of Surgery, HospitalTengkuBainun, Ipoh,Perak,Malaysia \\ ${ }^{3}$ Paediatric Surgery Unit,Department of Surgery, Hospital Raja \\ PerempuanZainabII,KotaBharu,Kelantan,Malaysia
}

\begin{abstract}
Introduction: Circumcisionis one of the commonest procedures done for medical or ritual reasons over the centuries. Currently, apart from traditional methods, circumcision is practiced in hospital under sterile conditions as well as in the community in a clean but nonsterile setting .Whereas the hazards of traditional circumcision have been extensively documented, the results of mass circumcision done in the community have not been objectively studied. This raises questions on the safety of such practice and whether improvements should be recommended to promote safety.

Objectives: This study aims to evaluate the differences in the incidence of the most common complications of bleeding and wound infection, when circumcision is done in the hospital and outside the hospital.

Methodology: This is a prospective study reviewing 75 circumcisions done in Hospital Raja PerempuanZainab II and 85 circumcisions done outside the hospital in the Kota Bharu and Pasir Mas district area between 1 November 2009 and 31 December 2009. Patients were followed up at one hour, three days and seven days after the circumcision. The incidence of bleeding and wound infection was recorded. Data analysis was done using SPSS software version 18.0.

Results: The incidence of wound infection is higher in the outside hospital group as compared to the inside hospital group (22.6\% vs 9.3\%,p 0.024), with a relative risk of 2.42. There is no significant difference in the incidence of bleeding between the outside hospital group and the inside hospital group (4.8\% vs 6.7\%, p 0.736).

Conclusion: This study proves that circumcision performed outside the hospital has more risk of developing wound infection compared to circumcision done in hospital. However bleeding complication remained identical in both groups .
\end{abstract}

Key words :Circumcision, Complications, Location

\section{Introduction}

Removal of the foreskin has been practised worldwide for various reasons from ancient times. Controversies exist regarding age at circumcision, indications and methods, personnel chosen to perform the procedure and location of the operation, whether in hospital or outside ${ }^{[1]}$. Whatever the circumstances the procedure is not free from complications ${ }^{[2]}$.

In Malaysia, during the long school holidays during November and December ,it is customary for ritual mass cicumcisions to be conducted in mosques,community halls, and school classrooms . The operations are done by trained Medical Assistants or Medical Officers under Local Anaesthesia . In such settings poor ventilation, poor temperature control and nonsterile environment is a feature. Occasionally, in more interior areas traditional circumcisions are done by Mudins who are mostly untrained but experienced ${ }^{[3]}$.

Comparisons have been made between outcomes of circumcisions done in hospital and traditional circumcisions done outside the hospital to study the complications and the results are widely reported elsewhere ${ }^{[4]}$. Bleeding and infection are the most common complications reported in the literature ${ }^{[5]}$. However, local reports are meagre in this regard. In view of the practice of large numbers of mass circumcisions done outside the hospital, and also in hospital, we designed a prospective study to study the incidence of bleeding and infection following circumcision in these two settings. It is hoped the results will help to improve the safety of this most important religious obligation. 


\section{Patients and Methods}

This is a prospective non randomised trial to study the incidence of bleeding and infection following circumcision done in the Raja PerempuanZainab Hospital II , Kota Bharu (HRPZ II )and in a community setting in the rural areas of KedaiBuloh and Bukit Tuku (outside hospital group ), between 1 Nov 2009 and 31 Dec 2009 . The study was approved by the Research and Ethics Committee,School of Medical Sciences ,Hospital UniversitiSainsMalaysia,KubangKerian,Kelantan. The protocol of the study was explained to the parents/guardians of the boys at the time of screening in the Outpatient Department of HRPZ II and at the community centres.

Included were all boys aged 7 to 12 years who needed ritual circumcision. Excluded were boys with a history of bleeding tendency,anatomical abnormality of the penis,evidence of local or systemic infection,medical disorders like nephrotic syndrome, Diabetes Mellitus or mental deficiency. Also if circumcision involved the use of diathermy, tissue glue or continuous instead of interrupted suture for haemostasis, or the use of any method other than Dorsal Slit (DS), such participants were excluded from the study.

In the HRPZ II group circumcision was performed in the operation theatre, whereas in the outside hospital group circumcision was done in nonsterile setting using non-sterilised gloves , with no proper ventilation and poor temperature control. In both groups, trained Medical Assistants with at least two years experience and Medical Officers conducted the circumcision. Chlorhexidine aqueous solution was used for preparing the genitalia and surrounding skin. Lignocaine plain solution (2\%) was used for Dorsal Nerve Block and Penile ring block anaesthesia (Max $3 \mathrm{mg} / \mathrm{Kg}$ ). After identifying the corona of the glans penis, and determining the extent of the slit two clamps will be placed from the meatal opening to within three fourths of the corona . The dorsal slit is made using scissors and the preputial skin is lifted perpendicularly and excised at its base leaving a cuff $0.5 \mathrm{cms}$ attached to the corona .Bleeding vessels are identified and ligated using plain $4 / 0$ catgut. Frenulum is approximated followed by the the approximation of cut preputial layers with interrupted plain $4 / 0$ catgut. After complete haemostasis $1 \%$ Chloramphenicol is applied without any dressings.

In both the groups the boys were observed for one hour post- circumcision for bleeding, before they were allowed home. Bleeding was defined as continuous oozing of blood at the wound site requiring further intervention like digital compression or re- exploration to control it. Those boys who reportedly bled after returning home were also considered as complicated .The first follow-up was on the third day postcircumcision. Wound infection was defined as purulent discharge from the wound site with at least one of the following : pain, tenderness, warmth, redness or swelling. In the outside hospital group the Medical Officer inspected the wound site for evidence of wound infection. Infected boys were given oral Cloxacillin, along with local dressings for the infected site. Participants in the inside hospital group were followed at the Outpatient Surgical clinic of HRPZ II . If infected similar treatment was given. Subsequent follow-up for both groups was done on the seventh post- circumcision day. If any boys were found with infection, in either group, the same treatment protocol was followed.

Data collected regarding bleeding, infection, besides age ,height, weight of the participants and their socio-economic background were entered into the proforma. Analysis was done using SPSS software (Version 12.0) . Means and Standard Deviation for continuous variables, and frequencies and percentages for categorical variables were calculated. Chi Square and Fisher test was used for association between categorical variables and Student $\mathrm{t}$ test for differences in means between the two groups. Generalised linear model was used to assess potential co- variates . Level of significance was set at $\mathrm{p}<0.05$.

\section{Results}

$159(58.67 \%)$ out of the total 271 boys recruited for the study attended follow-up and hence formed the study sample(Table 1). The parents/guardians of the boys circumcised outside the hospital were mostly fishermen and labourers $(80 \%)$, and were considered belonging to lower socio-economic status. Those who were circumcised in the hospital (HRPZ II ) belonged to the middle socio-economic status consisting of professionals and support staff of the Government and its agencies.

$151(94 \%)$ of the boys were aged between nine to twelve years . $8(6 \%)$ were aged below nine years( Fig.). Age and height were different in the inside hospital and outside hospital groups .However weight was identical(Table 2). The small difference in means of the height and age in the two groups was considered unlikely to influence the outcome of the study.

Wound infection was higher in the outside hospital group ( $\mathrm{p}=0.024$ ) (Table 3). Most of the infections were detected at follow-up on the third day. All of them were local infections without systemic manifestation. In the inside hospital group ,all seven infections resolved with oral antibiotic. Five boys with wound infection from the outside hospital group were managed in the surgical department of the HRPZ II as outpatient,which included oral antibiotic and wound dressings. By the second week all infections had cleared . No patient developed serious infection requiring admission for intravenous antibiotic. 
Incidence of bleeding in the inside hospital and outside hospital group was similar( $\mathrm{p}=0.763)$ (Table 4). Two boys each in the inside hospital group $(2.6 \%)$ and outside hospital group $(2.3 \%)$ developed bleeding within two days post circumcision. Exploration was successful. Bleeding was from penile vessels which had not been ligated or the ligatures had slipped . No blood transfusion was indicated. Exploration was done under local anaesthesia except for one apprehensive boy from the inside hospital group who required general anaesthesia . Other instances of minor bleeding were controlled by local compression

\section{Discussion}

This is the first prospective study done in Kelantan to compare the outcome of circumcision done inside the hospital and outside the hospital. We did not find any similar study done in Malaysia. However other authors elsewhere have reported on the outcomes of circumcisions done in hospital and outside hospital ${ }^{[6,7,8,9]}$.

We used the two proportion formula to calculate the sample size. Each group required eighty-five participants. For the inside hospital group seventy-five boys and for the outside hospital group eighty-four boys were recruited. Sixty percent from the outside hospital group and $57.3 \%$ from the inside hospital group attended the follow-up after circumcision. The rest were excluded from the study.

Majority of the boys circumcised were aged between nine and twelve years. Only $6 \%$ were below nine years. This trend was similar in both groups . Rashid in a cross -sectional study in the state of Kedah noted that nine years was the commonest age at circumcision. This is because in Malay culture most boys will be circumcised after their first recitation of the Holy Quran ${ }^{[3]}$. In the Philipines,most boys are circumcised at adolescence ${ }^{[10]}$. In the Republic of South Korea most boys (32\%) are circumcised between 6 and 11 years and by 18 years $90 \%$ of males had been circumcised ${ }^{[11]}$. Most Western countries notably North America ,Canada and Great Britain practice neonatal circumcision ${ }^{[12]}$. The Jews on the other hand circumcise their infants at day 8 after birth ${ }^{[13]}$.

We noted a mean difference of 9 months in age between the two groups in this study (10.12 vs $10.85, \mathrm{p}<0.001$ ). Also the mean difference in height was $6 \mathrm{cms}$. (130.48 vs $136.39, \mathrm{p}<0.001)$. We consider these factors unlikely to influence the outcome although they are statistically significant. Weight depends on nutrition which can influence wound healing and contribute to wound infection especially in children. In the present study we found the weight was evenly distributed among the two groups.(29.01 vs $31.13, p=0.142)$. We did not consider weight ,height and age as potential co -variates .

Bleeding $(0.1-35 \%)$ and wound infection $(0.2-0.4 \%)$ are the commonest early complications of circumcision $^{[5]}$. In this study we found circumcision done outside the hospital had a significantly higher risk of infection $(\mathrm{p}=0.024)$. Similar findings were reported by Atikeler and others ${ }^{[6,7]}$. However in Atikeler's retrospective study ,the outside hospital group were operated on by unlicensed circumcisers, and hence subject to reporting bias. In the present study sterile setting could not be achieved in the outside hospital group. But the method of circumcision ,namely Dorsal slit and the trained circumcisers were evenly matched in both groups. Naimer reported no wound infection in 200 neonatal circumcisions performed in hospital. However he notes that a strictly sterile environment is not a prerequisite for circumcision ${ }^{[14]}$. Ben Chaimprospectively reported a very low complication rate of $0.34 \%$ in 19,478 neonates circumcised in a nonmedical setting in the community ${ }^{[8]}$. This low incidence could be due to probable under-reporting as suggested by the author .

Increasing age has a greater risk for wound infection $(10 \%)$ when compared to neonates $(0.4 \%)^{[2]}$. The rich blood supply of the newborn penis and excellent capacity for wound healing could be a factor ${ }^{[8]}$. Difficulties in defining appropriate criteria for wound infection will also lead to variations in the reported incidence. According to CDC (1999) circumcision is considered clean surgery, with estimated risk of surgical site infection around $2.1 \%^{[15]}$. The source of infection could be from the environment, circumciser or from the patient ${ }^{[16]}$. The groin and perineal area have a higher bacterial load compared to other areas of the body. Gram negative species and anaerobes probably fecal in origin are to be expected. Although we used Chlorhexidine solution for skin preparation in both the groups, it is not effective against pseudomonas ${ }^{[17]}$, and to a lesser extent against Bacillus spores ${ }^{[18]}$. In the outside hospital group Chlorhexidine was also used for cleaning the instruments in between the circumcisions .

There was no significant difference in the incidence of bleeding between the inside hospital and outside hospital group ( $\mathrm{p}=0.736)$. Most bleeding stopped with local compression, with only $2.6 \%$ in the inside hospital group and $2.3 \%$ in the outside hospital group requiring re -exploration to control the bleeding . Atikelerreports significantly higher incidence of bleeding in the outside hospital group. This was probably because of the unlicensed and untrained circumcisers involved in his study ${ }^{[6]}$. According to Ozdemir, mass circumcision whether done in hospital or outside has a high risk of bleeding $(45.5 \%)$ and hence should be discouraged ${ }^{[7]}$. Training of medical personnel has a significant role in limiting such complications. This was amply demonstrated in a large prospective study done by Ahmed in the Comoros Islands ${ }^{[19]}$. In the present study both groups of circumcisions were performed by trained medical assistants with at least two years experience and also by medical officers. The method of circumcision is also important. We used the dorsal slit 
method, with ligation of bleeders when seen . In a 2008 study by Faris, the use of bipolar diathermy was shown to reduce significantly the incidence of bleeding, when compared with the dorsal slit technique in a hospital setting ${ }^{[20]}$.

We also studied the influence of socioeconomic status of the participants on circumcision. Most of the boys in the outside hospital group belonged to the lower socioeconomic classes consisting of farmers ,labourers or fishermen. In the inside hospital group the parents mostly were either Government support staff or professionals forming the middle socioeconomic category. This difference in status relating to education, attention to hygiene and nutritional status could potentially influence our results. However if the sampling method is taken into account we observe that the outside hospital group were recruited from the rural areas of Bukit Tuku and KedaiBuloh which is home to large population of fisherfolk, and labourers. Similarly the inside hospital group of participants were from Kota Bharu city which is the capital of Kelantan State and hence populated mostly by the middle socioeconomic category. In this study, the lower socio-economic group had a higher rate of infection $(19.1 \%$ vs $12.3 \%, \mathrm{p}=0.252)$. However it is not statistically significant (RR $1.56,95 \% \mathrm{CI}$ 0.7210-3.362 ). Similar findings were reported by Krieger from a prospective study in Kisumu District of Kenya. He concluded that circumcision could be safely done in a developing country with acceptable complication rate ${ }^{[21]}$. Even in a developed country like Netherlands, Schmitz was able to achieve acceptable results in a group of Muslim boys circumcised under local anaesthesia outside the hospital ${ }^{[9]}$. After univariate analysis we found when circumcision was done outside the hospital the relative risk for wound infection was 2.42( RR 2.42, 95\% CI 1.079-5.442) which is statistically significant. In the present study place of circumcision appears to be the only significant predictor of wound infection when results were analysed using generalised linear model for potential covariates (Table 5 ).

The present study is limited by the fact that $40 \%$ of the participants in the outside hospital group and $43.7 \%$ in the inside hospital group did not attend follow up. If we assume that these boys were free from complications then the incidence of bleeding and wound infection will be much lower. Our task was limited to the study of early complications, hence the short follow-up of seven days. It was not our objective to study late complications like foreskin related or disfigurement which will require longer follow-up. The strength of this study is also limited by the significant differences in the two groups with respect to age, height and the socioeconomic status .

\section{Conclusion}

The majority of boys circumcised in Kelantan were aged between eight to twelve years . Circumcision done outside the hospital in a nonsterile setting and environment had a significantly higher rate of wound infection compared to circumcision done inside hospital with a relative risk of 2.42. Bleeding complications were similar in the two groups. Ritual circumcisions will increase as the population grows. It is our duty as health care professionals to provide a safe environment to reduce the incidence of complications. Increasing the access to hospital, legislation and better education are options to be considered.

\section{Acknowledgement}

This study was supported by an Incentive grant from the Research wing of the UniversitiSains Malaysia. We also thank the participants and their parents and guardians, and also the Non Governmentalorganisations for their cooperation.

\section{Disclosure: The authors declare no conflict of interest.}

\section{References}

[1] MC Alanis, RS Lucidi.Neonatal circumcision: a review of the world's oldest and most controversial operation.Obstetrical\&Gynaecological Syrvey, 59(5), 2004,379-395

[2] N Williams, L Kapila. Complications of circumcision.British Journal of Surgery,80(10) ,1993,1231-1236.

[3] AK Rashid, TehSwee-Ping, KA Narayan. Traditional male circumcision in a rural community in Kedah, Malaysia.International eJournal of Science, Medicine and Education, 3(2), 2009, 19-23.

[4] HA Weiss, N Larke, D Halperin, I Schenker. Complications of circumcision in male neonates, infants and children: a systematic review. BioMed Central Urology ,10(2), 2010, 1-13.

[5] GW Kaplan. Complications of circumcision, Urological Clinics of North America ,10(3), 1983, 543-549.

[6] MK Atikeler, I Gecit, V Yuzgec, O Yalcin. Complications of circumcision performed within and outside the hospital.InternationalUrology and Nephrology,37(1), 2005, 97-99.

[7] E Ozdemir. Significantly increased complication risks with mass circumcisions. British Journal of Urology, 20, 2004, $136-139$.

[8] J Ben Chaim, PM Livine, J Binyamini, B Hardak, D Ben-Meir, Y Mor. Complications of circumcision in Israel: a one year multicentre survey. The Israel Medical Association Journal, 7(6), 2005,368-370.

[9] RF Schmitz, TW Schulpen, JC van Wieringen, M Kijlstra, EJ Verleisdonk, C van der Werken [Good results from circumcision of Muslim boys performed outside the hospital][Dutch]. NederlandsTijdschriftvoorGeneeskunde, 143(12), 1999, 627-639.

[10] RB Lee. Circumcision practice in the Phillipines: community based study. Sexual Transmission and Infection, 81(1), $2005,91$.

[11] DS Kim, JY Lee, MG Pang. Male circumcision : a South Korean perspective. British Journal of Urology International, 83,1999, Suppl 1:28-33. 
[12] WHO/UNAIDS: Male circumcision: global trends and determinants of prevalence ,safety and acceptability. World Health Organisation 2008

[13] SA Rizvi, SA Naqvi, M Hussain, AS Hassan. Religious circumcision: a Muslim view. British Journal of Urology International, 83 , 1999, Suppl 1:13-16.

[14] SA Naimer, A Trattner. Are sterile conditions essential for all forms of cutaneous surgery? The case of ritual neonatal circumcision.Journal of Cutaneous Medicine and Surgery, 4(4), 2000,177-180.

[15] CDC : Guideline for prevention of surgical site infection .Centers for Disease Control and Prevention, 1999

[16] W Whyte, AHambraeus, UG Laure, J Hobom. The relative importance of routes and sources of wound contamination during general surgery. Journal of Hospital Infection ,18(2), 1999, 93-107.

[17] DW Burdon, JL Wmitby. Contamination of hospital disinfectants with Pseudomonas species. British Medical Journal, 2,1967,153155.

[18] SP Gorman, DS Jones, AM Loftus.Thesporicidal activity and inactivation of Chlorhexidinegluconate in aqueous and alcoholic solution. Journal of Applied Microbiology ,63(2), 1987, 183-188.

[19] A Ahmed. Childhood circumcision : a planned approach. Tropical Doctor,37(4), 2007, 239-241.

[20] MM Fariz, MN Tarmizi, MH Ainani, AM Khairil, AFaizal, I Sagap. A prospective randomised comparison of bipolar diathermy versus conventional dorsal slit technique for ritual circumcision : a Malaysian experience. La ClinicaTerapeutica, 162(2), 2011, 543-546.

[21] JN Krieger, RC Bailey, JC Opeya, BO Ayieko, FA Opiyo, K Agot,et al. Adult male circumcision : results of a standardised procedure in Kisumu District,Kenya. British Journal of Urology International

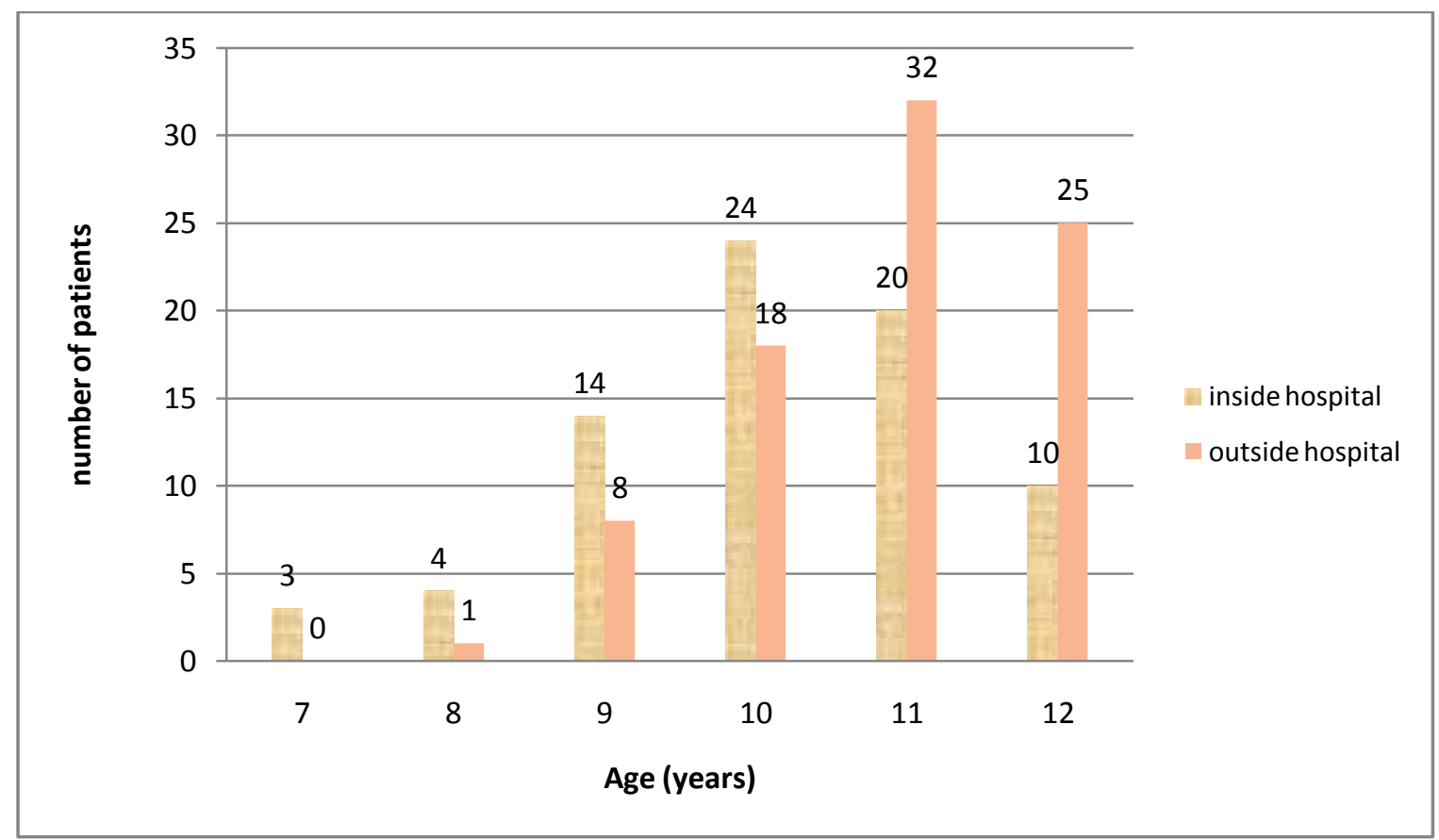

Fig. Age of the participants in the two groups

Table 1. Samples recruitment in inside hospital and outside hospital group

\begin{tabular}{lcccc}
\hline $\begin{array}{l}\text { Place of circumcision } \\
\text { Circumcisions } \mathrm{n}\end{array}$ & follow-up n & Number of & Patients attended & Percent \\
\hline Inside hospital & & & 75 & 57.30 \\
Outside hospital & KedaiBuloh & 131 & 65 & 72.20 \\
& Bukit Tuku 50 & 90 & 38.00 & 58.67 \\
\hline Total & & 271 & 159 & \\
\hline
\end{tabular}

Table 2.Age,weight and height characteristic in between inside hospital and outside hospital groups

\begin{tabular}{lllc}
\hline Variables & Place of circumcision & Mean (SD ) & $\mathrm{p}^{\mathrm{a}}$ \\
\hline Age & Inside hospital & $10.12(1.25)$ & $<0.001$ \\
& Outside hospital & $10.85(0.99)$ & 0.142 \\
\hline \multirow{2}{*}{ Weight } & Inside hospital & $29.01(7.83)$ & \\
& Outside hospital & $31.13(9.98)$ & \\
\hline Height & Inside hospital & $130.48(8.50)<0.001$ & \\
& Outside hospital & $136.39(9.97)$ &
\end{tabular}

\footnotetext{
${ }^{\text {a }}$ Independent $\mathrm{t}$ test
} 
Table 3.Incidence of wound infection between the two groups

\begin{tabular}{llllll}
\hline $\begin{array}{l}\text { Groups } \\
\mathrm{n}(\%)\end{array}$ & $\mathrm{n}$ & No infection & Infection & $\mathrm{X}^{2}$ Test $(\mathrm{df})$ & $\mathrm{P}$ \\
\hline Inside hospital & 75 & $68(90.7 \%)$ & $7(9.3 \%)$ & & \\
& & & & & \\
Outside hospital & 84 & $65(77.4 \%)$ & $19(22.6 \%)$ & & 0.024 \\
\hline
\end{tabular}

\begin{tabular}{lcccc}
\hline & \multicolumn{4}{c}{ Table 4. Incidence of bleeding between the two groups } \\
\hline $\begin{array}{l}\text { Groups } \\
\mathrm{n}(\%)\end{array}$ & $\mathrm{n}$ & No bleeding & Bleeding $\mathrm{X}^{2}$ test $(\mathrm{df})$ & $\mathrm{p}$ \\
\hline Inside hospital & 75 & $70(93.3 \%)$ & $5(6.7 \%)$ & $0.736^{\mathrm{a}}$ \\
Outside hospital & 84 & $80(95.2 \%)$ & $4(4.8 \%)$ & \\
\hline
\end{tabular}

${ }^{\mathrm{a}}$ Fisher exact test was used for the calculation

Table 5. Association between risk factors for wound infection using Generalised Linear Model ${ }^{\mathrm{a}}$

\begin{tabular}{lcccc}
\hline $\begin{array}{l}\text { Variable } \\
\text { risk }\end{array}$ & Relative & p-value & \multicolumn{2}{c}{$95 \%$ Confidence Interval } \\
& & & Upper & Lower \\
\hline Place of circumcision & 2.64 & 0.052 & 0.99 & 7.03 \\
Socio-economic status & 1.15 & 0.763 & 0.46 & 2.88 \\
\hline
\end{tabular}

${ }^{\mathrm{a} C a l c u l a t i o n}$ using Stata 9.2 software 